\title{
Tsafon
}

Revue d'études juives du Nord

$77 \mid 2019$

Contribution à l'histoire des traductions juives de la Bible hébraïque

\section{Le dernier mot, inventaire}

Récit de l'alyah d'une rescapée

des camps de Theresienstadt et Auschwitz

\section{Eva Erben}

\section{OpenEdition}

\section{Journals}

Édition électronique

URL : https://journals.openedition.org/tsafon/2004

DOI : $10.4000 /$ tsafon.2004

ISSN : 2609-6420

Éditeur

Association Jean-Marie Delmaire

Édition imprimée

Date de publication : 1 septembre 2019

Pagination : 143-164

ISSN : $1149-6630$

\section{Référence électronique}

Eva Erben, « Le dernier mot, inventaire », Tsafon [En ligne], 77 | 2019, mis en ligne le 16 septembre 2019, consulté le 25 juin 2021. URL : http://journals.openedition.org/tsafon/2004 ; DOI : https:// doi.org/10.4000/tsafon.2004 


\title{
Édition
}

\section{Le dernier mot, inventaire}

\author{
Récit de l'alyah d'une rescapée \\ des camps de Theresienstadt et Auschwitz
}

Eva Erben ${ }^{*}$

\section{Présentation par Anne Karila}

Née à Děčín (Tchécoslovaquie) en 1930, Eva Erben a passé son enfance à Prague. Fin 1941, elle et ses parents sont déportés à Theresienstadt et trois ans plus tard, à Auschwitz.

Son père $\mathrm{y}$ est assassiné, sa mère meurt durant une des «marches de la mort » qui suivent l'évacuation du camp, au début du printemps 1945. Eva en réchappe miraculeusement et est recueillie par une famille de paysans de Postřekov, les Jahn, dont le nom figure désormais sur le mur des Justes parmi les nations à Yad Vashem, à Jérusalem. Après la guerre, elle retourne à Prague et suit une formation d'infirmière. En 1949, elle et Peter, son futur mari, émigrent en Israël.

CZECH REPUBLIC
ČESKA REPUBLIKA
ANDELOVA-WEISS KARLA
BABOR FRANTISEK \& MARIA
BACOVSKY LEOPOLD \& ANTONIE
BARANCOVA MARIA
BINDER-URBANOVA ANNA
BLEHA JOSEF
CHLUP ANNA \& JAROSLAV
FIERZ OLGA
FISERA JOSEPH
FRYDL KAREL
HAJEK MILOS
HAJKOVA ALENA
HOLUB ALOIS
HONDIKOVA MARIA \& VLADIMIR
HREKOW ANDRZEJ \& WIERA
JAHN KRISTOF \& LUDMILA
MUY dES JUSTES, Yad VAShEM
JÉrUSAlEM

* Traduction de l'hébreu par Danielle Delmaire, Université de Lille, SHS, et Anne Karila, Université de Lille. La présentation originelle du texte hébreu a été respectée, pour l'essentiel. Les modifications ou rectifications ont été opérées avec l'accord d'Eva Erben. Les notes sont des traductrices. 
De sa déportation, Eva n'a commencé à parler qu'en 1979, sollicitée par l'institutrice de son fils Amir. Son récit couché sur papier est paru en Israël en 1988 : אמה ספרי לי, את היית שם [Maman raconte-moi, tu étais là-bas]. Plusieurs traductions et éditions commentées ont suivi (en Tchéquie, Allemagne, France, Angleterre) et donné lieu à des émissions de radio et de télévision ainsi qu'à plusieurs films. Mais surtout, Eva n'a pas cessé d'aller à la rencontre d'enfants et de jeunes, en Israël et dans plusieurs pays d'Europe, pour leur raconter son histoire.

J'ai fait la connaissance d'Eva en 1999, à l'occasion d'une de ces rencontres organisée par l'Institut Goethe de Lille. Elle m'a tout de suite " chargée » de traduire son récit de l'allemand (Oubliée, souvenirs d'une jeune fille juive, Paris, L'école des loisirs, 2001, réédition 2018) et au fil des ans, notre amitié s'est muée en profonde affection.

Quand au printemps 2018, Eva m'a mis entre les mains ce deuxième manuscrit, Inventaire, avec un laconique «Traduis-le en français et publie-le », l'urgence de dérouler ce nouveau fil de mémoire s'est imposée. Même si à 88 ans, Eva continue à conduire sa voiture avec panache et à vaquer à ses activités, le temps presse: la plupart des rescapés sont morts, et Peter est parti, lui aussi, en 2017, à 97 ans.

Juste après le décès de Peter, Eva a ressenti le besoin de retracer en détails le périple de leur émigration et leur installation en Israël. Ces quelques pages montrent comment, jeunes immigrants rescapés de la Shoah, seuls et démunis, ils ont pu recommencer à vivre.

Ceux qui ont rencontré Eva lui ont largement manifesté leur reconnaissance. Elle a conservé dans une grosse malle d'osier quantité de lettres, dessins d'élèves, articles de divers pays, travaux de recherche etc. On la remercie « d'éclairer le monde et les cœurs ». Alors quand le doute pointe, parfois - qui s'intéressera à tous ces documents lorsqu'elle ne sera plus là puisque ses enfants ne lisent pas l'allemand ni le tchèque, ni le français ou l'italien ? -, c'est ce qu'elle retient. En se souvenant, elle veut parler d'espoir, de lumière.

Merci infiniment à Danielle Delmaire d'accueillir ce témoignage dans Tsafon et d'avoir effectué une première traduction du texte hébreu, que j'ai pu retravailler ensuite avec les éclairages d'Eva, en passant par notre langue de communication qu'est l'allemand. Autant de pérégrinations humaines et détours linguistiques pour apporter une nouvelle pierre à l'édifice jamais achevé de la mémoire. 


\section{Récit d'Eva Erben}

Mars 1949

Le Transylvanie s'approche de la côte de Haïfa.

Le soleil couchant se reflète sur les toits, la mer scintille.

Je suis émue par ce spectacle. Est-ce bien réel ?

Est-ce ici que je vais poursuivre ma vie ? Ici que va naître l'enfant que je porte ? Si j'en crois l'agitation dans mon ventre, lui aussi est ému.

Peter et moi nous tenons enlacés sur le pont et il me murmure : « Ici, je bâtirai une maison pour toi, entourée d'un jardin avec des fleurs. Nous y aurons des enfants qui ne connaîtront pas la douleur et la méchanceté des hommes »).

Il y a encore quatre ans, nous nous battions pour rester en vie et nous avons eu de la chance : nous avons survécu... Nous sommes rentrés chez nous avec l'intention de continuer, de reprendre tout comme avant. Mais la réalité fut différente.

Tout avait disparu.

Le 15 mai 1948 fut proclamé l'État d'Israël.

J'ai rencontré Peter à la communauté juive, où nous étions réunis pour écouter la proclamation du nouvel état juif par Ben Gourion.

Ensuite nous sommes allés faire la fête au Café Mánes, sur les rives de la Moldau. Nous avons dansé, nous nous sommes aimés et ne nous sommes plus quittés.

Soixante-huit années ont passé. Je suis dans la maison que Peter a bâtie, comme il l'avait promis. J'écris, je me souviens... Et je songe à quel point notre vie a été marquée par la politique.

À ce moment-là, en 1948, c'est la prise du pouvoir par les communistes en Tchécoslovaquie qui gâchait nos projets. Il fallait chercher un autre endroit. Nous étions envahis par la crainte. Nous voulions vivre, mais nous avions peur de l'avenir, il avait lui aussi une odeur d'antisémitisme, c'était à nouveau effrayant. Durant toutes ces années de guerre passées dans le ghetto de Theresienstadt ${ }^{1}$, nous avions rêvé du jour où nous pourrions rentrer chez nous, à Prague, et reprendre

\footnotetext{
${ }^{1}$ Theresienstadt : Mis en place par les nazis dans l'ancienne ville de garnison de Terezín et présenté à l'opinion internationale comme un ghetto modèle, Theresienstadt était un camp de concentration et de transit avant la déportation vers les camps d'extermination de l'Est.
} 
notre vie là où elle s'était arrêtée. Notre espoir s'est heurté à une autre réalité. L'invasion soviétique était incompatible avec un avenir en Tchécoslovaquie. Nous comprenions que nous n'avions rien à attendre de ce régime. Nous avons beaucoup réfléchi et finalement décidé de quitter la Tchécoslovaquie, nous n'avions pas le choix. Mais cette décision entraînait de nombreuses difficultés: avoir un permis de sortie, s'informer sur nos droits et faire les démarches pour obtenir les papiers exigés.

Nous ne savions pas bien encore si nous voulions aller en Australie ou en Israël.

Dans le petit appartement de Peter à Prague, nous avons commencé nos préparatifs: il fallait se procurer l'autorisation de quitter la Tchécoslovaquie, les attestations et autres documents. Durant cette période, la bureaucratie était à son comble. Nous avons reçu une liste de ce que nous pouvions emporter et nous nous sommes limités à la quantité de bagages et d'objets autorisés, comme par exemple: 2 paires de chaussures, 6 paires de chaussettes...

Peter a sorti une valise noire, assez élégante et a dit : « Mon père a acheté cette valise en 1918 en Italie, après sa libération de captivité, et ma mère, avant de partir pour le ghetto de Theresienstadt en 1940, 1'a donnée à Fania, notre bonne. Elle y avait fourré quelques vêtements et des draps, pensant que nous en aurions besoin en revenant chez nous, après la guerre $»$.

Fania avait rendu à Peter la valise avec tout son contenu et, en 1948 , je la remplissais à mon tour pour voyager vers une destination inconnue.

En tout cas, cela fut vite fait, car nous n'avions pas grand-chose. Moi je n'avais même pas de manteau...

La somme d'argent autorisée était limitée elle aussi, or nous ne savions pas combien de temps nous devrions attendre à Paris, ni de quoi nous allions vivre d'une manière générale. Quelle serait notre destination, finalement? Israël, l'Australie ? Comment subvenir à nos besoins en attendant?

Dans les journaux, on a beaucoup écrit à propos de la destruction de Berlin et, entre autres, de la seule usine qui fournissait à toute l'Europe les épingles spécialement destinées à la conservation des papillons rares. 
Il se trouve qu'à Opava, en Moravie, un petit atelier produisait les mêmes épingles. Cela avait attiré l'attention de Peter et finalement nous en avons acheté quelques boîtes pour les emporter et les vendre à Paris.

Un jour, nous avons appris que le Museum d'histoire naturelle de Paris, où travaillait le baron Stephan von Breuning, cherchait désespérément des épingles pour conserver des papillons rares qu'un scientifique avait rapportés d'Afrique... Une simple annonce dans le journal.

Les petites boîtes attendaient dans un coin avec nos vêtements. Bien sûr, on n'était pas autorisé à sortir ce genre de choses de Tchécoslovaquie. Nous avons réfléchi à la façon de les emballer afin que les policiers, aux frontières, ne les remarquent pas si par hasard ils ouvraient notre valise.

Un autre objet pour le moins curieux attendait d'être emballé : un pot de chambre. En voici l'histoire :

Comme je n'avais pas 18 ans, j'avais un tuteur, le docteur Erwin Wintermintz. Un de ses amis, le docteur Kurt Wehle, luttait en faveur de la restitution des biens juifs confisqués par les nazis. Le gouvernement soviétique de l'époque fit pression sur lui pour qu'il abandonne ses actions. Kurt n'a pas cédé et il a même intenté un procès contre les institutions. Bien sûr, ce ne fut pas du goût du gouvernement, qui émit un ordre d'emprisonnement à son encontre. Or, une ancienne connaissance de Kurt apprit qu'il serait arrêté au petit matin. Le jour même, Kurt, sa femme et leur bébé âgé d'un an sont sortis de chez eux, prétextant qu'ils allaient se promener. Ils ont gagné Wilsonovo Nadráží, la gare centrale de Prague $^{2}$, et sont montés dans un train qui les a emmenés directement à Paris. Sans valise, afin de ne pas éveiller de soupçons. Ils craignaient d'être suivis.

C'est pourquoi il ne s'agissait plus pour nous à présent d'emporter seulement des vêtements, mais aussi des langes et ce pot de chambre qui attendait d'entrer dans la valise.

Alors j'ai soudain eu l'idée de mettre les épingles à l'intérieur du pot, entourées des couches, du petit linge de corps et de nos chaussettes. J'avais presque fini quand Peter est arrivé. Il a regardé et dit: «Formidable ! ", puis il a disparu un instant, pour réapparaître avec un drapeau tchèque que nous conservions dans l'armoire. Il en a enveloppé tout le contenu de la valise et l'a fermée.

\footnotetext{
${ }^{2}$ De 1945 à 1948, la gare centrale de Prague était appelée Gare Wilson.
} 
Le lendemain matin, nous avons quitté Prague, sommes montés dans un train pour Paris, la peur au ventre, ne sachant pas ce qui nous attendait. Deux jours auparavant, j'avais découvert que j'étais enceinte. Le train s'est ébranlé et notre regard a suivi les maisons, les arbres, les lacs qui défilaient derrière la vitre. Nous étions tous les deux silencieux, plongés dans nos pensées, peut-être pensions-nous aux mêmes choses. À la frontière, le train s'est arrêté. Nous avons entendu les pas lourds des policiers dans le couloir, les portes qui claquaient, - angoisse...

Vint le moment où un policier a pointé le doigt vers notre valise. Peter l'a descendue et l'a ouverte... Je ne respirais quasiment plus, j'observais le policier. Il a vu le drapeau puis nous a regardés, les larmes aux yeux. Il a dit : « Tout va bien », et il est parti.

Peter a remonté la valise et m'a regardée, il m'a prise dans ses bras et serrée très fort. Il a murmuré : « šst'astnou cestu », bon voyage.

Septembre 1948, Paris

Nous avons débarqué le lendemain matin à la gare de l'Est, avec notre valise, un peu fatigués et effrayés par tout le bruit autour de nous. Où aller, maintenant ?

Nous sommes passés devant un magasin de légumes plein de couleurs et d'odeurs. Émerveillé par les bananes, Peter a promis : «Quand on aura de l'argent, on s'en achètera ». Nous n'avions jamais vu un régime de bananes...

En poursuivant notre chemin, nous avons trouvé un hôtel ; il n'était pas particulièrement engageant, mais nous y sommes quand même entrés et on nous a donné une chambre au cinquième étage, sans ascenseur.

Peter a traîné la valise, la chambre était déprimante.

Il a dit: "Ça ne fait rien, on va passer la nuit ici et demain on verra. Viens, allons chercher le musée et vendre des épingles au baron pour avoir de l'argent ». (Il pensait aux bananes...)

Nous nous sommes rafraîchis dans la chambre, puis nous sommes ressortis.

Une fois à nouveau dans la rue bruyante, $\mathrm{j}$ 'ai été prise de vertige et soudain, tout est devenu noir autour de moi, je me suis écroulée.

J'ai repris connaissance dans le bistro d'à côté devant une tasse de café et un croissant, - je ne sais toujours pas comment Peter a fait pour payer tout cela. 
Il souriait et m'a dit sur un ton de reproche : «Qu'est-ce que tu me fais ? Je parle, je parle et tu ne réponds pas », - une réaction typique de mon bien aimé tout puissant ${ }^{3}$.

Nous avons trouvé le musée ainsi que le baron, qui nous a bien reçus et acheté quelques épingles. En nous raccompagnant à l'hôtel, il nous a raconté qu'il appartenait à la famille des Habsbourg, mais qu'il avait été rejeté par les siens parce que la femme qu'il avait épousée n'était pas une aristocrate. Alors il était devenu professeur, spécialiste des papillons et directeur du département des recherches au Museum d'histoire naturelle de Paris. Ses travaux étaient pointus.

Quand il a vu où nous logions, il a dit : « Vous ne pouvez pas rester ici ». Avec résolution, il a annulé notre chambre et nous a amenés à l'Hôtel de Suez sur le boulevard de Strasbourg, où il habitait avec sa femme, Martha.

Durant tout notre séjour à Paris, les von Breuning ont été pour nous un couple d'amis formidables. Nous avons trouvé en eux des gens qui s'intéressaient à notre sort ; peut-être voyaient-ils en nous les enfants qu'ils n'avaient jamais eus. Martha veillait notamment à me faire manger toutes sortes de coquillages et de poissons, et j'étais subjuguée par la manière dont le baron fumait sa cigarette $\left(\right.$ Gauloise) ${ }^{4}$ : il ne la retirait jamais de sa bouche et la cendre ne tombait pas.

Kurt et Hanna Wehle nous ont reçus dans leur petit appartement à Rueil-Malmaison, à quelques dizaines de kilomètres de Paris, un logement que le Joint ${ }^{5}$ leur avait fourni ; Kurt avait en outre aussitôt obtenu un poste à la communauté. Ils ont été très contents de récupérer le pot de chambre, et la petite Eva nous a tout de suite montré qu'elle savait l'utiliser.

Kurt et Hanna avaient la quarantaine ; leur union était pour eux deux un second mariage. Ils étaient l'un et l'autre arrivés mariés au ghetto de Theresienstadt, mais leurs conjoints n'avaient pas survécu.

Ils nous paraissaient donc assez âgés. Hanna s'est immédiatement aperçue que j'étais enceinte et, apprenant que je n'avais pas vu de médecin depuis notre départ de Prague, elle a arrangé rapidement une consultation chez le docteur Nerson. Gratuitement, par amitié, celui-ci a surveillé ma tension pendant plusieurs mois.

\footnotetext{
${ }^{3}$ Souligné par Eva.

${ }^{4}$ Écrit entre parenthèses en lettres latines « Guloas ».

${ }^{5}$ Le Joint ou American Jewish Joint Distribution Committee est une organisation humanitaire d'assistance sociale juive des USA. Fondé en 1914, il est venu en aide aux juifs d'Europe pendant la guerre puis d'Israël.
} 
J'ai pris l'habitude d'aller tous les vendredis acheter une carpe et une hala $^{6}$ rue des Rosiers, dans le quartier juif, puis de me rendre en train chez Hanna, à Rueil-Malmaison. Là, j'ai aussi apprécié les bains chauds, un véritable luxe à l'époque. À l'hôtel, nous n'avions qu'un lavabo.

Chez Hanna, j'ai retrouvé l'atmosphère de la maison de mes parents, qui avait disparu et que j'avais presque oubliée. Les attentions et l'amitié d'Hanna étaient pour moi comme l'eau pour une fleur flétrie. Une amitié presque maternelle.

Kurt et Peter rentraient en fin de journée et dans la pièce à tout faire, la table était déjà mise, avec la hala que j'avais achetée dans le quartier juif. Nous parlions, évoquions des souvenirs et tirions des plans sur la comète jusque tard dans la nuit, avant que le dernier train nous ramène de Rueil-Malmaison à Paris.

Le 31 octobre 1948, nous nous sommes mariés à la mairie du $X^{\text {ème }}$ arrondissement, à Paris.

Comme cadeau de mariage, Kurt et Hanna nous ont offert des billets pour l'opéra Ä̈da de Verdi.

Soixante-huit ans sont passés et aujourd'hui, en regardant en arrière, je suis émue : je nous vois montant les escaliers de l'Opéra, la lumière des lustres en cristal étincelait, il y avait une atmosphère de fête.

Pendant quelques heures, nous avons quitté un monde dans lequel nous n'avions aucun avenir sûr et nous nous sommes beaucoup amusés, en particulier lorsque sont apparus sur scène deux éléphants vivants. Je revois tout cela comme si c'était hier. Nos journées, durant cette période, étaient remplies d'attente, de décisions à prendre, de dilemmes, de doutes.

Comment et où commencer une nouvelle vie ? En Israël, où l'on combattait, où la sécurité était précaire ? En Australie, où l'antisémitisme sommeillait, mais existait bel et bien ?

Les États-Unis, l'Amérique ne nous attiraient vraiment pas. Les lettres d'Israël que nous écrivait Pavel, le frère de Peter qui avait immigré avec 1'Alyat Hanoar ${ }^{7}$, étaient pleines d'espoir : avec les Arabes, tout allait se terminer et il nous attendait, il avait une petite cabane, un coin pour nous. Nous pourrions habiter chez lui.

\footnotetext{
${ }^{6}$ Pain de Shabbat.

${ }^{7}$ Alyat Hanoar ou alyah des jeunes est une organisation sioniste créée en 1933 pour aider des jeunes juifs à émigrer en Palestine. À la fin des années 1930, elle s'est surtout investie dans le sauvetage de jeunes juifs fuyant le nazisme d'Europe centrale. Puis elle s'est occupée de l'accueil d'orphelins de la Shoah en Israël.
} 
Mon ventre grossissait. Où le petit être qui se développait dans mon corps allait-il naître ? Il fallait se décider, le temps pressait.

« Nous irons en Israël», avons-nous tranché... «Advienne que pourra!»

Nous avons vendu mon visa pour l'Australie 70 dollars. Avec cet argent, Peter a acheté des billets de première classe pour le bateau Kedma. Il était prévu que le bateau quitte le port de Marseille le 6 février.

Durant les deux mois qui nous restaient, nous avons profité de cette ville fantastique qu'est Paris. Nous avons découvert la richesse de son histoire, ses trésors artistiques, le Louvre, nous avons visité tous les endroits merveilleux du Paris de cette époque. Notre décision d'en finir avec la vie de migrants nous avait soulagés. Nous avons vendu toutes les épingles et fait nos adieux à tout le monde. Le couple von Breuning a tout fait pour nous convaincre de rester en France. Ils ont même voulu nous acheter une petite maison, dans les environs de Paris. Ils étaient tellement opposés à ce que nous partions en Israël... Mais nous ne pouvions pas accepter leur proposition.

Cette fois, le contenu de notre valise était différent : pas d'épingles, ni de langes, mais un grand sous-verre protégeant des papillons rares aux couleurs formidables, que le baron nous avait offert pour nous remercier de lui avoir procuré des épingles; ce cadre a longtemps orné notre logement en Israël.

Ainsi le 4 février, tôt le matin, le train est parti pour Marseille. À notre arrivée, le soleil brillait, il faisait beau; nous sommes allés directement au bureau d'embarquement et là, une surprise désagréable nous attendait. Nous regardant avec étonnement, l'employée nous dit : «Vous étiez inscrits pour le bateau précédent ».

Le bateau avait pris la mer deux jours plus tôt. Son départ avait été anticipé à cause de la météo. "Pourquoi ne nous avez-vous pas avertis ? », a demandé Peter. Il n'a pas obtenu de réponse... Ils avaient oublié...

Nous étions abasourdis.

« Je vous inscris pour le bateau qui part le 6 mars, le Transylvanie, c'est un meilleur bateau ", dit la dame pour nous consoler. Mais qu'allions-nous faire pendant un mois encore ? Nous n'avions pas d'argent? Nous n'avions rien!

Elle nous rassura : " On va vous héberger au camp de réfugiés de Bandol, vous pouvez y aller. » 
Nous sommes restés une nuit à Marseille. D'abord nous sommes passés à la poste et avons envoyé un télégramme à Kurt, à Paris, pour qu'il nous fasse parvenir rapidement un peu d'argent. Puis nous avons trouvé un hôtel sur le boulevard Garibaldi, l'Hôtel de la Rose.

Nous avons déposé la valise dans la chambre et sommes allés au port, plein de marins et d'odeurs bizarres. Nous nous sommes installés à une table et avons commandé une bouillabaisse. Nous n'avons pas beaucoup parlé. Au milieu du repas, Peter a dit : « Demain matin, on va à Bandol ».

Nous étions subjugués par le port, la mer... C'était ma première rencontre avec la mer. Nous n'avons fait que regarder, respirer et manger. Puis nous sommes rentrés à l'hôtel, fatigués et nous nous sommes endormis rapidement.

$\mathrm{Au}$ milieu de la nuit, la pluie et des coups de tonnerre m'ont réveillée, je me suis levée pour fermer la fenêtre et au moment où j'atteignais le volet branlant, il s'est décroché et est tombé du troisième étage sur les escaliers, dans un formidable fracas.

Il n'y avait personne en bas. Peter dormait.

Je suis retournée au lit, à côté de lui, sous la couverture que nous partagions, je l'ai tirée sur notre tête, je ne voulais pas voir, je ne voulais pas entendre, seulement dormir.

Le lendemain, nous avons pris l'autobus jusqu'à Bandol. De loin, nous avons aperçu un camp près de la mer, sur le sable, des baraques... Nous nous sommes approchés, avons examiné les alentours.

Peter a posé la valise et a dit : « Reste là, je vais voir ». Je l'ai suivi des yeux jusqu'à ce qu'il disparaisse dans un des bâtiments. Il en est ressorti rapidement et m'a fait des signes de la main. Revenu à ma hauteur, il a dit : «C'est un camp de concentration amélioré ».

Nous avons marché vers le village.

Il était constitué de petites maisons, près de la mer, et sur plusieurs d'entre elles était accroché un panneau « Chambre à louer ».

Peter a sonné à une porte et une femme est apparue. Elle a demandé : "Vous voulez louer une chambre ? Entrez, je vous en prie ».

La chambre paraissait satisfaisante. Un lit blanc, une fenêtre avec vue sur la mer, une table et deux chaises. Un rideau fleuri qui s'agitait à la brise venue de la mer.

Peter a demandé : « Combien coûte la chambre ? » Jusqu'à ce jour j'entends encore la réponse : «Cinq francs ». Est-ce que c'était à la semaine, au mois ou à la journée ? Je ne m'en souviens plus. 
Nous étions fatigués, affamés, et la chambre était si belle.

La femme nous a laissés et, au bout d'une vingtaine de minutes, elle est revenue avec deux assiettes de soupe, des tranches de pain et une carafe d'eau.

« Mangez, bon appétit», a-t-elle dit en souriant. Visiblement, elle avait compris à qui elle avait loué la chambre.

Là, durant tout un mois, nous avons passé du bon temps en compagnie de pêcheurs. Peter a même essayé de pêcher en suivant leurs conseils. Un jour, il a ramené une étoile de mer que nous avons enduite de vernis à ongles transparent; nous l'avons emportée en Israël et la valise a bien pué...

Maintenant, en me remémorant ces journées, je me dis que nous avons eu beaucoup de chance de rater le bateau précédent. Ainsi, pendant un mois de cette période particulière, nous avons bien vécu, même si, ne sachant pas ce que nous réservait l'avenir, nous n'avons pas pu en profiter pleinement.

Comme notre subsistance ne nous coûtait rien puisque les pêcheurs nous donnaient du poisson, Peter a eu une idée : «Que dirais-tu de passer notre lune de miel sur la Côte d'Azur? C'est tout près d'ici». Nous allions voir des lieux où nous n'aurions jamais imaginé nous rendre. Il m'est difficile de raconter aujourd'hui comment nous avons fait tout cela. Ce ne fut pas un voyage organisé avec réservations à l'hôtel, mais un simple périple en bus avec quelques haltes. Notre premier arrêt fut Cannes, nous y sommes arrivés au moment du fameux carnaval et avons été émerveillés à la vue des gens qui se réjouissaient si spontanément. Nous avons découvert un monde étincelant ; les hôtels sur la Promenade des Anglais à Nice, nous ne les connaissions qu'à travers les romans.

À Monte Carlo, Peter a introduit une petite pièce dans une machine à sous et à notre stupéfaction, elle a recraché quelques francs. Peter a dit : « C'est comme ça que ça se passe, au début on gagne, on est séduit, et après on perd $»$.

Nous nous sommes attardés un petit moment puis avons continué vers Menton, où nous avons vu pour la première fois des oranges sur les arbres. Peter m'en a cueilli une. J'ai conservé la pelure et l'ai retrouvée dans mon sac, à Haïfa.

Au bout d'une semaine, nous sommes rentrés à Bandol. Près de la mer, nous avons rencontré une famille qui attendait elle aussi le Transylvanie. Ils avaient une fille de 5 ans, nous avons sympathisé. Il 
s'est avéré qu'il s'agissait du Dr Pelzman, gynécologue. Quelle chance inouïe : j'avais un médecin pour le voyage.

Le hasard a voulu que le $1^{\text {er }}$ mai 1949, quand j'ai donné naissance à notre fille Dany, à l'hôpital Rambam de Haïfa, c'est lui qui m'a accouchée.

Nous avons exploré toutes les ruelles de Bandol.

Thomas Mann, Franz $\mathrm{Werfel}^{8}$, y avaient trouvé refuge après l'arrivée des nazis au pouvoir.

Nous aussi, nous y avons trouvé refuge...

Le 6 mars, nous avons quitté Bandol pour Marseille et embarqué sur le Transylvanie, qui a pris la mer le jour même. La sirène du bateau a déchiré l'air, comme pour dire adieu... Oui, adieu à l'Europe.

Nous avions une cabine rien que pour nous, en première classe puisque nous avons payé le voyage en vendant mon visa pour l'Australie, ce qu'il était alors possible de faire pour se procurer un peu d'argent.

Les machines se sont mises en marche. Puissant bruissement de la mer... Voilà, nous étions partis.

Le deuxième jour du voyage, il y a eu une tempête. De hautes vagues inondaient le pont. Dans la salle à manger, les tables, qui avaient été fixées au sol par des chaînes, se sont détachées et ont valsé d'un bout à l'autre de la pièce.

Cela faisait un bruit terrible. Les gens commençaient à devenir verts. Peter a dit que le meilleur endroit où se tenir, dans ces cas-là, c'était la proue du bateau, et il fallait faire en sorte que les mouvements $\mathrm{du}$ corps suivent ceux du bateau. Mais là, les hautes vagues nous ont presque trempés, alors nous sommes vite retournés dans notre cabine.

Le bateau tanguait comme une coquille de noix. Dans notre cabine, nous avions des couchettes superposées. Allongée en bas, je me cramponnais au châlit. Peter a ouvert la valise et en a sorti toutes ses cravates. Je me suis demandé : «Mon Dieu, dans cet enfer, qu'est-ce qu'il a besoin de cravates ?»Il les a liées les unes aux autres par des nœuds qu'il avait vraisemblablement appris à faire chez les scouts, de manière à former une longue corde avec laquelle il m'a attachée à la couchette en déclarant : "Comme ça tu ne rouleras pas ». Et moi, je me suis dit que si le bateau sombrait, je serais prisonnière du lit. Alors une

\footnotetext{
${ }^{8}$ Comme de très nombreux écrivains et artistes en exil, Thomas Mann et Franz Werfel sont passés par Bandol avant de s'installer à Sanary-sur-mer; en 1940, la plupart de ces exilés qui n'avaient pas encore quitté la France furent internés au camp des Milles, d'où un grand nombre fut déporté vers les camps de la mort.
} 
pensée m'a envahie : après tous les efforts que nous avions faits pour rester en vie et après avoir survécu à la marche de la mort, nous allions mourir noyés dans un naufrage en mer.

Pendant soixante-huit ans, j'ai conservé au fond de moi le souvenir de cet événement, et toutes ces années, quand quelque chose me contrariait, j'y repensais.

Le lendemain, la tempête était complètement passée. Le ciel était dégagé, la mer calme, on avait installé des chaises longues sur le pont. Il était difficile de croire que le cours de la vie puisse changer aussi vite.

Le 11 mars, dans l'après-midi, le bateau a jeté l'ancre.

Il soufflait un vent chaud, caressant et fatigant : le hamsin. Autour de nous, ça parlait et ça criait dans une langue que nous ne comprenions pas. Finalement, un homme s'est approché et nous a demandé : « Taxi ? » Ça, nous l'avons compris... Oui, partir d'ici, vite.

La route n'était pas agréable, nous étions silencieux. Le conducteur s'est arrêté et nous a abandonnés sur un chemin de terre.

C'était la bonne adresse, Pavel attendait dehors. L'instant se chargea d'électricité : dix années s'étaient écoulées depuis que les deux frères s'étaient quittés, et le monde avait changé.

Où est l'appartement? Où Pavel habite-t-il ? Je ne vois pas de maisons. Je vois une immense caisse en bois. Pavel nous y emmène.

Un lit de l'Agence juive ${ }^{9}$, une table et deux chaises... En guise d'armoire, quatre clous au mur, auxquels sont pendus les vêtements de Pavel. Pas d'eau, un puits dehors... Pas de toilettes, dehors, ça aussi...

Plus tard, j'ai compris que cette caisse était un de ces conteneurs que les émigrants ayant réussi à quitter l'Allemagne avaient emportés avec eux, remplis de meubles. Ces caisses avaient ensuite été transformées en habitations.

La nuit est tombée soudainement, les deux frères se sont placés à la " porte de la maison » et se sont replongés dans le passé. Les questions étaient sans fin.

Je me suis installée sur l'unique lit et j'ai écouté. J'ai fini par comprendre que même si Pavel n'avait pas vécu la Shoah, il n'avait pas pour autant été épargné. Tout était tellement triste.

Je me suis blottie dans ce lit qui piquait, j'ai caressé mon gros ventre et pleuré en silence.

\footnotetext{
${ }^{9}$ L'Agence juive est une organisation sioniste créée en 1929 qui se destinait à devenir l'exécutif de l'Organisation sioniste mondiale en Palestine. Elle prit en charge les différentes migrations de juifs vers la Palestine puis vers l'État d'Israël.
} 
Au milieu de la nuit, j'ai senti Peter s'installer à côté de moi dans le lit étroit de l'Agence juive.

Premier matin en Israël : le soleil brille, le ciel est bleu, sans un nuage. Pavel nous a laissé un mot: «Je suis allé acheter des petits pains $»$.

Nous nous sommes lavés au puits, dans la cour, et Pavel est revenu avec des petits pains, de la halwa ${ }^{10}$ et trois pots de yaourt pour moi, un délice !

Nous n'avions pas terminé notre petit déjeuner que Jacques faisait son apparition. C'était le mari de Nourit, la cousine de Peter, il était arrivé dans le pays en $1938^{11}$, avait fait les guerres, ici, et servi dans l'armée ; depuis, il était instructeur.

Jacques et Nourit vivaient dans un logement social à Kyriat Hayim, au nord de Haïfa. Jacques venait nous chercher car, vu mon état, il estimait que nous ne pouvions pas rester chez Pavel ; il nous hébergerait, en location.

À cette époque, trouver un appartement était difficile. Israël n'était pas encore en mesure d'intégrer le grand nombre de nouveaux immigrants.

$\mathrm{Au}$ bout de trois mois environ, nous avons eu la chance d'obtenir une pièce chez des amis qui avaient pu emménager dans un logement social. Notre nouvelle adresse était : Kyriat Hayim, Tet Vav 14, chez les Sternberg. Leur fille, Daliah, plus jeune que moi de quelques années, était pianiste. Elle se produisit plus tard en soliste dans tout le pays ${ }^{12}$. Je l'avais entendue lors d'un concert qu'elle avait donné à Prague.

Peter a trouvé du travail au port dans un chantier naval, chez Kirchstein Grünspan.

Dans notre pièce, il y avait un petit coin pour la cuisine, mais nous partagions les commodités et la salle de bains avec le propriétaire. Il fallait beaucoup d'efforts, de patience et de compréhension pour surmonter ces difficultés, mais nous étions contents de notre sort.

Enfin seuls, tous les trois, Peter, moi et notre petite Dany qui venait de naître. Avec son premier salaire, 70 livres israéliennes, Peter a acheté deux chaises à un Arabe et, sur le chemin, il a trouvé quatre briques qui ont servi de pieds de table. La table, c'était notre chère valise. Peter l'a installée sur les briques, je l'ai recouverte d'un foulard en guise de nappe.

\footnotetext{
${ }^{10}$ La halwa est une douceur turque à base de crème de sésame avec des pistaches.

11 « Dans le pays » : c'est-à-dire en Palestine sous mandat britannique.

${ }^{12}$ Israël, cette fois-ci.
} 
Nous avions deux lits de fer, que nous avait donnés l'Agence juive, avec des matelas qui piquaient. Un parc, cédé par un de nos proches, servait de lit à notre bébé.

Avec ce premier salaire, nous avons eu aussi très à cœur de rembourser notre dette envers Kurt de Paris. Nous sommes restés en relation avec lui et sa femme toute notre vie. Lorsqu'ils ont émigré à New York, nous leur avons rendu visite, et Amir, notre plus jeune fils, a eu l'honneur de perdre contre Kurt aux échecs.

Un jour, Kitti Koretz, une amie de Prague, est venue nous voir. Avant la Seconde Guerre mondiale, ses parents étaient propriétaires d'une mercerie renommée qui vendait des boutons, du fil, des lacets.

Après la guerre, Kitti était revenue de Theresienstadt avec sa mère et son frère. Leur bel appartement de Prague les attendait. Nous y avons passé des dimanches pluvieux à jouer au Monopoly. Le frère de Kitti est ensuite devenu commentateur sportif à la radio et était très apprécié des auditeurs.

En entrant chez nous à Haïfa, Kitti a balayé notre unique pièce du regard et j'ai compris qu'elle n'était pas à l'aise.

Avec étonnement, elle a demandé : "C'est tout ce que vous avez? » Moi, je me sentais bien, à ce moment-là nous avions plus ou moins fini de nous installer et disposions de tout ce dont nous avions besoin. Je n'ai donc pas réagi à sa remarque.

Kitti a continué : «Avec ma mère, nous habitons à Tel-Aviv. Nous avons une pièce chez un oncle ». Cet oncle était en fait l'homme d'affaires Yerushalmi, assez connu dans le pays pour sa production de cordes.

« Nous avons apporté de Prague un conteneur avec tous nos meubles, il attend dans le port de Jaffa car nous n'avons pas de place, et nous payons chaque mois une consigne. Vous voudriez peut-être quelques meubles? »"

C'est ainsi qu'un beau matin, un camion est arrivé rue Tet Vav, avec deux fauteuils, un divan recouvert de velours vert, une très belle table en verre, un lampadaire et un buffet. J'étais étourdie par tant de choses. Comment faire rentrer dans notre petite pièce tout ce luxe qui, de toute façon, avec ce climat chaud, ne nous convenait pas ?

Considérant l'ensemble, Peter a réfléchi et essayé de tout caser au mieux. La pièce était surchargée, or moi je rêvais de meubles « Lacol », alors à la mode, une sorte d'équivalent d'Ikea de maintenant. 
Dans la pièce encombrée de meubles massifs, on ne pouvait plus bouger. Avec son optimisme habituel, Peter me rassurait : «Attends que nous ayons une maison normale, tout va s'arranger $»$.

Mais la «maison normale » était loin, à des années-lumière de nous.

Nous nous sommes inscrits pour obtenir un logement à Kyriat Hayim. L'inscription coûtait 300 livres, que Pavel nous a données, et nous avons suivi les travaux de construction avec impatience. Chaque shabbat, nous nous frayions un chemin dans le sable, avec la poussette de Dany, afin de voir l'avancée des travaux.

Jusqu'à ce que nous découvrions, au bout d'un certain temps, que le travail n'avançait plus. Tout avait été abandonné...

L'entrepreneur, Monsieur Druker, était parti à New York. Il n'avait pas tenu parole et nous, nous avions perdu l'argent.

Cela aussi, il nous a fallu le surmonter.

Peter a décidé de chercher un travail qui nous permettrait de nous payer un logement. Aussi s'est-il tourné vers Solel Boneh ${ }^{13}$ à Tel-Aviv, il était prêt à accepter n'importe quel emploi et même à quitter Haïfa.

On l'a envoyé à Beer-Sheva. Il y soufflait un violent hamsin. Un sable fin venu du désert remplissait l'air, cela nous était inconnu. À cette époque, il n'y avait ni végétation ni arbustes pour retenir le sable, et les gens souffraient de ces tempêtes. Peter a estimé qu'il ne pouvait pas faire vivre sa famille dans un tel endroit et il a abandonné l'idée de s'installer à Beer-Sheva.

Il est donc retourné chez Solel Boneh. Là, on lui a conseillé de prendre un emploi à Ashkelon, ville où l'on était en train d'implanter une usine de tuyaux, la célèbre «Yuval Gad», afin d'irriguer le Néguev.

Dans le cadre de son travail, on nous a promis un logement, dans une cabane en bois préfabriquée, bien sûr.

Durant un mois et demi, je suis restée seule avec Dany à Haïfa, pendant que Peter travaillait chez Yuval Gad et vivait en célibataire dans un immeuble.

Chaque jour, nous attendions avec impatience de recevoir une carte de Peter. Il n'y avait pas encore de téléphone. La séparation a été pénible, depuis notre départ de Prague, nous ne nous étions pas quittés.

\footnotetext{
${ }^{13}$ Solel Boneh est la plus ancienne et l'une des plus grandes entreprises de construction et de génie civil en Israël.
} 
Le $1^{\mathrm{er}}$ mai 1951, nous avons déménagé de Haïfa à Ashkelon, le jour même des deux ans de Dany. Elle tenait dans ses mains une poupée de chiffon, son cadeau d'anniversaire.

Yohanan, de Yuval Gad, nous a emmenés de Kyriat Hayim à Ashkelon dans un petit camion du type International, chargé de nos meubles. Le voyage a duré six heures ${ }^{14}$.

En 1951, Ashkelon avait vraiment un aspect arabe.

$\mathrm{Au}$ bout de la colline, il y avait huit cabanes de Yuval Gad, la moitié d'une cabane pour une famille.

Nous nous sommes installés avec Uri et Aviva Gazit qui étaient arrivés avec l'autre véhicule de l'usine, conduit par Shaoul. Aviva était enceinte de Yaron, à un stade avancé ; à Haïfa, nous étions passés rue Herzl pour qu'elle récupère une robe de grossesse rouge.

Uri était né à Mexico de parents yekke ${ }^{15}$, il parlait donc allemand. Aviva, sa femme, était une tsabra ${ }^{16}$, native du pays, et elle ne parlait qu'hébreu. C'est avec elle que j'ai appris l'hébreu.

Chez nous, il y a toujours eu à manger. Avec les vieilles halot de shabbat, je confectionnais des croquettes. Certaines choses, j'avais appris à les faire pendant la guerre... Et quand il n'y avait pas de pommes de terre ni de riz, je préparais des knödel avec de la farine : on peut tirer quelque chose de rien... La recette n'était pas très connue. Un jour que nous étions encore dans les cabanes, j'ai invité Uri et Aviva et celle-ci s'est émerveillée sur la nourriture. Elle a voulu savoir comment je préparais la pâte. Je n'ai pas compris le mot «pâte ». Alors Aviva a pris dans ses mains un peu de farine qu'elle a mélangée avec de l'eau et, en tendant ses mains vers moi, elle m'a dit : «Voilà de la pâte ! »

La cabane sentait le bois naturel, elle n'était pas peinte. Pour tout dire, nous n'avions pas l'électricité ni l'eau courante. Nous allions puiser l'eau à un puits dans les environs, et l'électricité nous provenait d'un générateur que l'usine avait mis à notre disposition.

Après quelque temps, tout s'est arrangé et nous étions très contents.

Nous n'avions pas de réfrigérateur, j'entends bien réfrigérateur et non pas frigidaire. Aviva s'est extasiée devant nos meubles mais elle a été surprise que nous n'ayons pas réfrigérateur, c'était tellement

\footnotetext{
${ }^{14}$ Pour environ 150 kilomètres.

${ }^{15}$ Yekke: terme péjoratif désignant un juif immigré d'Allemagne et pétri de bonnes manières.

${ }^{16}$ Tsabra : terme hébreu qui désigne un juif né en Palestine ou en Israël. Le mot signifie «figue de Barbarie », le tsabra est comme la figue, piquant à l'extérieur mais doux à l'intérieur.
} 
indispensable, par ces températures. Elle a immédiatement fait de la place pour nous dans le sien; je lui ai rappelé ce geste jusqu'à la fin de sa vie.

Aviva avait découvert l'épicerie d'Alfonso... un bel homme. Dans sa boutique, il y avait de bonnes choses et on trouvait toujours tout. Un jour que j'y étais, avec Dany dans sa poussette, celle-ci s'est mise à pleurer. Pendant que je la consolais en tchèque, une jeune femme que je ne connaissais pas est entrée, elle m'a souri et a dit: « Tu es de Tchécoslovaquie? » C'était la femme d'Alfonso, le Marocain. Elle venait d'arriver de Tchécoslovaquie, était blonde aux yeux bleus, et enceinte. La petite fille qui leur naquit, Oli, est devenue bien des années plus tard l'institutrice d'Amir.

Notre médecin était le docteur Krause de Berlin, marié à Rubi, une Arabe chrétienne du Liban.

La population d'Ashkelon était très variée. Lors d'une visite chez des amis d'Aviva par exemple - lui était officier de l'armée -, j'ai eu envie d'aller aux toilettes et avec stupéfaction j'y ai trouvé, en guise de papier, des pages de Guerre et paix de Tolstoï.

Nos amis restés à Haïfa étaient loin de nous. Nous, nous étions dans le Néguev et pour eux, le mot Néguev était effrayant. Il y faisait chaud, c'était près de la frontière... donc un peu dangereux. Aucun ne venait nous voir.

Yuval Gad produisait des tuyaux pour irriguer le Néguev, et ce n'est que lorsque le réseau local a été ouvert que les amis nous ont rendu visite.

En 1953, notre famille s'est agrandie : notre fils Alon est né dans la cabane.

Petit à petit, nous avons nourri l'espoir d'avoir bientôt une véritable maison à nous.

Au bout de deux ans environ, on a commencé à construire des logements sur les hauteurs d'Ashkelon, à Afridar, qui était jusqu'alors un terrain sablonneux. Mais comme nous n'avions pas d'argent pour nous inscrire en vue d'obtenir un de ces logements, Peter a décidé d'acheter un terrain et de bâtir lui-même. Ainsi, nous avons demandé un prêt de 900 livres à la banque. Pendant des années, j'ai apporté chaque mois cinq livres à Monsieur Adivi, le directeur de la banque d'Ashkelon.

Uri et Aviva habitaient déjà dans un des premiers immeubles qui avaient été construits. Un jour, nous avons été invités à l'anniversaire de leur fils Yaron et nous nous y sommes rendus avec nos voisins Shaya et 
Guerda, et leurs filles, dans le pick-up de Yuval Gad. Au retour de la fête, notre véhicule a essuyé des coups de feu, les quatre pneus étaient crevés ; à l'endroit où se trouve l'actuelle agence de location de voitures « Eldan », Guerda, moi et les enfants sommes sortis du pick-up et rentrés aux cabanes, tandis que Shaya et Peter allaient à la police avec les soldats qui roulaient derrière nous et avaient été légèrement blessés. Après avoir fait la déclaration à la police, ils sont partis vers l'hôpital Kaplan de Rehovot.

Cette nuit-là, le réservoir d'eau du kibboutz Yad Mordechaï ${ }^{17} \mathrm{a}$ explosé. Nous avons vu l'explosion depuis la terrasse de la cabane.

En écrivant tout cela maintenant, je nous revois jeunes, mais avec les yeux d'une femme âgée. Nous étions des enfants. Si seuls... sans nos parents, sans aucune aide. Toujours si seuls, toute la vie...

Afin d'acheter des matériaux, Peter a pris une avance de six mois sur son salaire et, avec cela, il s'est procuré du bois, des briques et tout le nécessaire pour débuter la construction.

Pour que nous puissions acheter à manger, j'ai accepté, le cœur lourd - je voulais tellement rester auprès de mes enfants - , de travailler comme infirmière dans le service public, dans les localités nouvellement fondées du Hevel Lakhish ${ }^{18}$, où arrivaient les habitants. J'étais infirmière pour ces gens-là.

Peter a commencé à construire seul. Pour les travaux spécifiques de professionnels, il a employé des ouvriers.

Le shabbat, nous sortions tôt le matin, avant la chaleur. Toute la famille se rendait à pied des cabanes jusqu'à notre maison à Afridar, pour arroser le béton... Pour que cela avance... Même notre petit garçon savait déjà tenir un mètre ruban.

Au début de l'année scolaire 1957, nous avons quitté la cabane pour notre maison rue Harimon. Les meubles y ont trouvé leur place.

Peter avait accompli la promesse qu'il m'avait faite à bord du Transylvanie: «Ici, quelque part, nous construirons une maison où naîtront nos enfants ».

En 1970, nous est né un autre fils, Amir.

\footnotetext{
${ }^{17}$ Yad Mordechaï, à $10 \mathrm{~km}$ au sud d'Ashkelon, est un kibboutz très proche de la frontière avec la bande de Gaza. Il se trouvait en première ligne face aux Égyptiens lors de la guerre d'Indépendance et eut à subir d'importants dommages.

${ }^{18}$ Hevel Lakhish est la région autour de la ville de Kiryat Gat, entre les monts de Judée et Ashkelon.
} 


\section{Inventaire en photos}

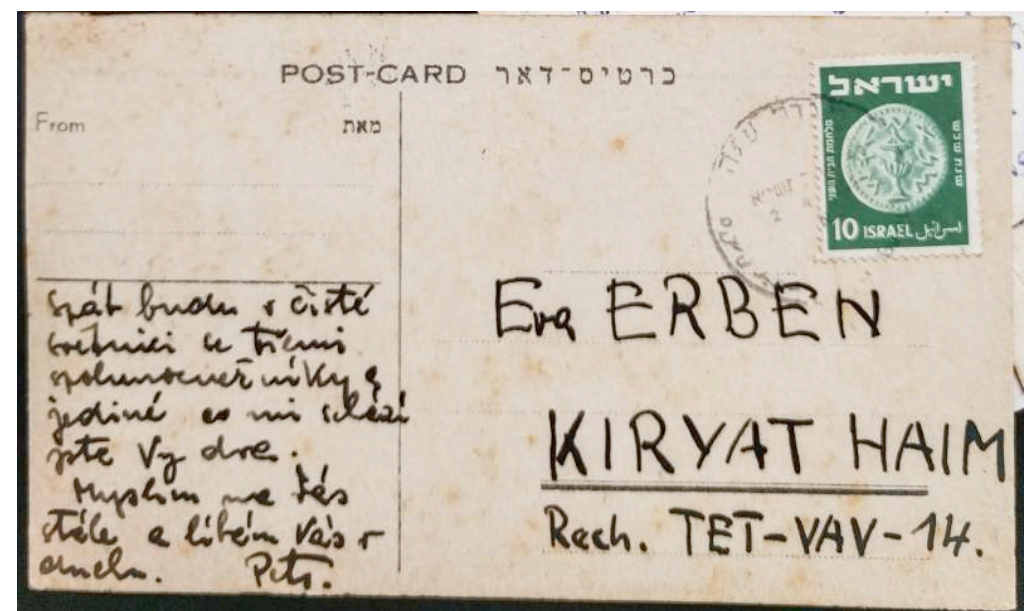

Carte envoyée par Peter (voir la signature) à Eva Erben à son adresse, 14 rue Tet Vav à Kyriat Haïm, près de Haïfa

« Chaque jour, nous attendions avec impatience de recevoir une carte de Peter»

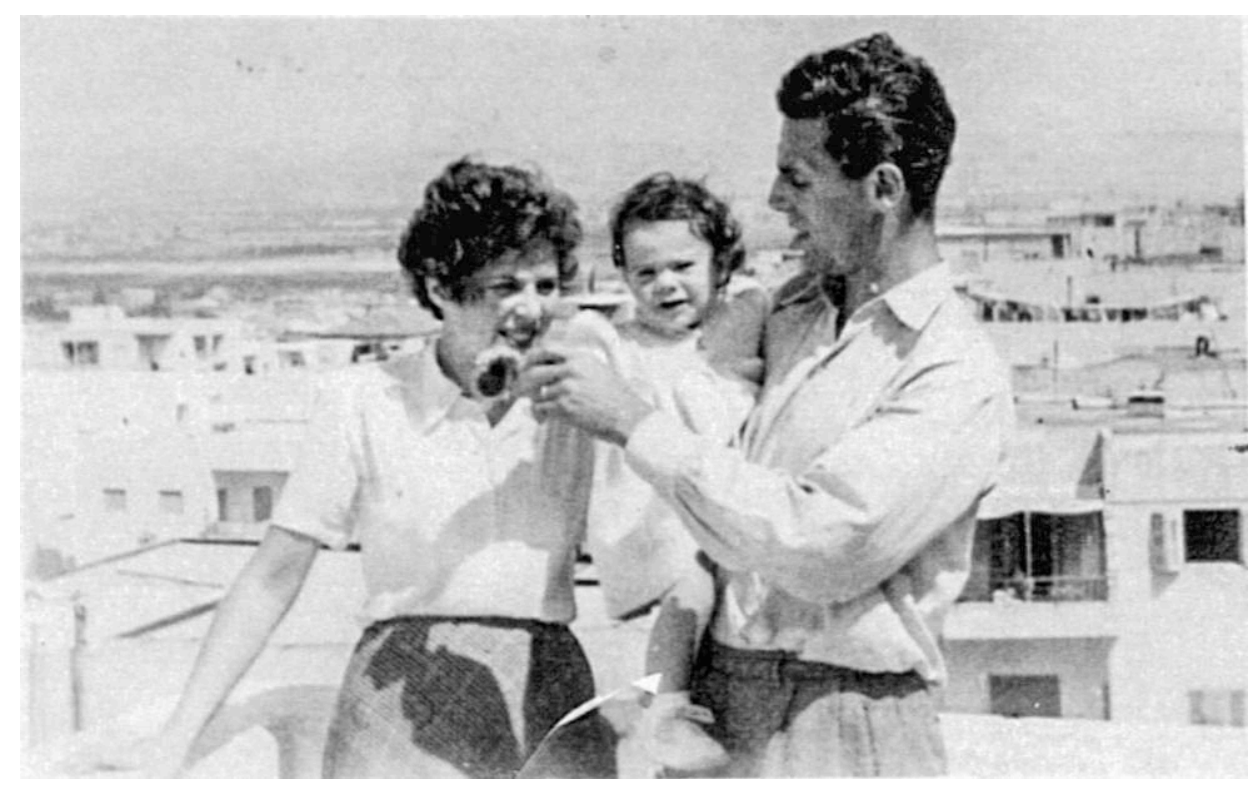

Eva et Peter avec la petite Dany et sa poupée de chiffon, cadeau pour l'anniversaire de ses deux ans 
Eva Erben et sa famille devant la maison d'Ashkelon, en construction

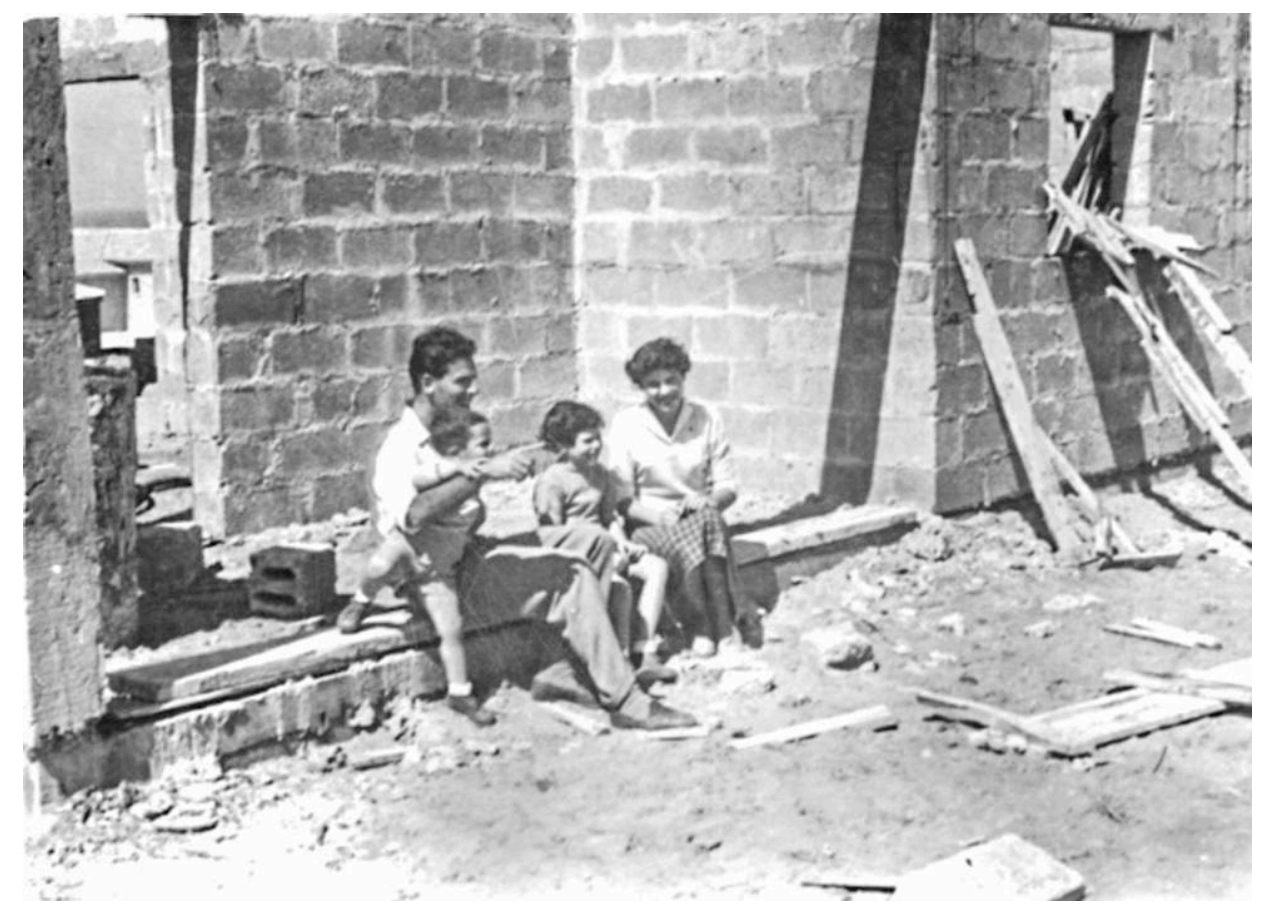

Peter avec Alon, Dany et Eva.

« Le shabbat, nous sortions tôt le matin, avant la chaleur. Toute la famille se rendait à pied des cabanes jusqu'à notre maison à Afridar, pour arroser le béton... Pour que cela avance... Même notre petit garçon savait déjà tenir un mètre ruban » 


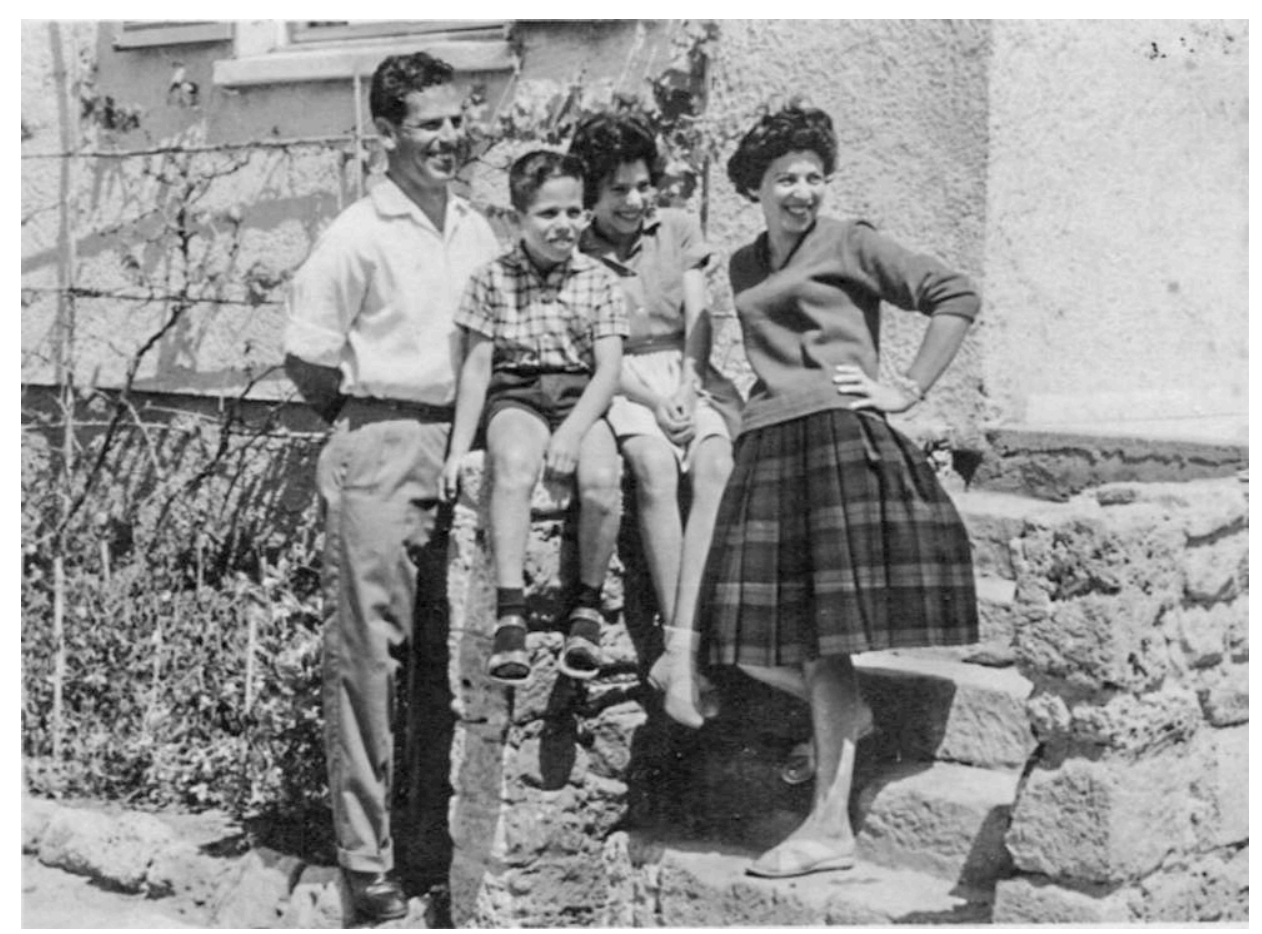

Peter, Alon, Dany et Eva

Dany est née le $1^{\text {er }}$ mai 1949, à l'hôpital Rambam de Haïfa

«En 1953, notre famille s'est agrandie: notre fils Alon est né dans la cabane »

«En 1970, nous est né un autre fils, Amir » 